\title{
Hamiltonians Defined as Quadratic Forms ${ }^{\star}$
}

\author{
BARRY SIMON \\ Fine Hall, Princeton University
}

Received June 1, 1970

\begin{abstract}
We present a complete mathematical theory of two-body quantum mechanics for a class of potentials which is larger than the usual $L^{2}$-classes and which includes potentials with singularities as bad as $r^{-2+\varepsilon}$. The basic idea is to define $H_{o}+V$ as a sum of quadratic forms rather than as an operator sum.
\end{abstract}

\section{§ 0. Introduction}

After one has established the connection between quantum mechanics and Hilbert space objects [1], a host of nontrivial mathematical questions arise. These involve establishing the self-adjointness of the Hamiltonian, studying qualitative properties of bound states, investigating the question of the existence of the limits needed for scattering theory, and proving the physically expected properties of the $S$-matrix. For the two-body case, $H=-\Delta+V$, with $V$ in one of the four classes, $L^{2}+L^{\infty}, L^{2}+\left(L^{\infty}\right)_{\varepsilon}{ }^{1}$, $L^{2}, L^{2} \cap L^{1}$ there is a fairly complete theory of these questions [2] going back to the famous paper of Kato [3]. Our purpose here is to extend this theory to a larger class of potentials than the $L^{2}$ classes. While one can establish some $n$-body results for these larger classes (e.g. Hunziker's theorem on the position of the continuum [4] goes through), we only discuss the two-body case here - a case for which a complete theory exists. A fuller discussion of this theory can be found in [5]; in this note we wish to emphasize the main results and the physics behind these results.

Before describing the classes we treat in detail, let us explain why such larger classes should exist. Consider a potential $V=r^{-\alpha}$. This is in an $L^{2}$ class only if $0 \leqq \alpha<3 / 2$ but physically there is nothing singular about $-\Delta-r^{-3 / 2}$. It is only at $\alpha=2$ that singular things begin to happen. At $\alpha=2$, the uncertainty principle "proof" that $H$ is bounded below breaks down (and in fact $-\Delta-c r^{-2}$ is not bounded below if $c>1 / 4$ ).

* Based on a thesis submitted to Princeton University in partial fulfillment of the degree of Doctor of Philosophy.

${ }^{1} X+\left(L^{\infty}\right)_{\varepsilon}=\left\{f \mid(\forall \varepsilon) f=x_{\varepsilon}+g_{\varepsilon}\right.$ with $\left.x_{\varepsilon} \in X ;\left\|g_{\varepsilon}\right\|_{\infty}<\varepsilon\right\} ;$ e.g. $r^{-1} \in L^{2}+\left(L^{\infty}\right)_{\varepsilon}$ on $\mathbb{R}^{3}$. 
There are indications that a quantum mechanical particle in an $r^{-2}$ force field "falls into the origin" ([6], pp. 118-121) and these indications are borne out by a detailed investigation of the Feynman Path Integral for such a potential [7]. In summary, one expects there to be an extended theory which will include potentials $r^{-\alpha} ; 3 / 2 \leqq \alpha<2$. In fact, using special properties of central potentials, one can already establish a great deal of physics for such potentials [8].

In producing such a generalized theory, the first thing we must give up is the method of defining $-\Delta+V$ as a sum of operators on $D(-\Delta)$, for Strichartz [9] has shown a multiplication operator $V(x)$ has $D(V)$ $\supset D(-\Delta)$ if and only if $V$ is "uniformly locally $L^{2}$ " in which case $-\Delta+V$ is self-adjoint on $D(-\Delta)$ by Kato's theorem [3]. To find a suitable substitute for definition as an operator sum, let us return to the basic quantum mechanical meaning of the statement $C=" A+B$ " for observables $A, B, C$. Given the physical interpretation for "observable", we should demand only that $\langle\psi, C \psi\rangle=\langle\psi, A \psi\rangle+\langle\psi, B \psi\rangle$, i.e. interpret the sum in an expectation value sense. Thus letting $Q(A)=\left\{\psi \mid\langle\psi, A \psi\rangle\langle\infty\}^{2}\right.$, we will attempt to define $H=-\Delta+V$ as follows. Define " $H$ " as a quadratic form on $Q(\Delta) \cap Q(V)$ and then show this quadratic form is the quadratic form of some self-adjoint operator. Such a procedure has already been suggested by Nelson and Faris [10] (and applied to the case $V \in L^{3 / 2}$ ). A related technique can be found in [43].

We will deal with the classes, $R+L^{\infty}, R+\left(L^{\infty}\right)_{\varepsilon}, R, R \cap L^{1}$ where $R$ is the set of $V$ 's obeying

$$
\|V\|_{R}^{2}=\int|x-y|^{-2}|V(x)||V(y)| d^{3} x d^{3} y<\infty .
$$

This condition has been independently isolated by several authors [11] and studied, although not systematically, by several others [12]. In Appendix 1, we study the relation of this Rollnik condition ${ }^{3}$ to other conditions; we remark that the class $R+L^{\infty}$ is nearly maximal in the class of potentials for which $Q(-\Delta) \subset Q(V)$ and for which $-\Delta+V$ can be defined as a sum of forms and that $r^{-\alpha}(0 \leqq \alpha<2) \in R+L^{\infty}$. The property that distinguishes $R$ from other conditions one might pick is that $V_{1 /}^{1 / 2}\left(H_{o}+E \pm i 0\right)^{-1} V^{1 / 2}$ is an integral operator with Hilbert-Schmidt kernel if $V \in R .^{4}$

After discussing the definition of the Hamiltonian in $\S 1$ we turn to establishing qualitative properties of bound states in $\S 2$. Our main tools in this study are a certain integral equation and the approximation of $V$ with $L^{2}$ potentials. In $\S 3$, we discuss the existence and unitarity of the

${ }^{2}$ In terms of spectral projections this means $\int|\lambda| d\left\langle\psi, E_{\lambda} \psi\right\rangle\langle\infty$.

${ }^{3}$ We name the condition after the individual who was the first to isolate it (at least to our knowledge).

${ }^{4}$ Let $V_{\|}^{1 / 2}(x)=|V(x)|^{1 / 2} ; V^{1 / 2}(\mathrm{x})=V(\mathrm{x}) / V_{\|}^{1 / 2}(x)=V_{\|}^{1 / 2}(x)[\operatorname{sgn} V(x)]$. 
$S$-matrix. As one might expect the difference between the $R$ and $L^{2}$ classes which is a difference in finite singularities is not important; in fact, one major result is that once one can prove existence and unitarity for bounded potentials with a certain behavior at $\infty$, one can prove existence and unitarity for locally- $R$ potentials with the same behavior at $\infty$. Finally, in $\S 4$ we establish eigenfunction expansions in the sense of Ikebe [13] when $V \in R \cap L^{1}$.

Let us try to summarize what we regard as the major result of the note. Because of simple physical arguments and because of Khuri's work on forward dispersion relations [8], most physicists regard a potential as singular only if it has a singularity as bad as $r^{-2}$. On the other hand, people who concern themselves with foundational questions ${ }^{5}$ often set the line only at $r^{-3 / 2}$. Our goal here is to bring the two standards into line at $r^{-2}$. All that changes at $r^{-3 / 2}$ is the interpretation of $-\Delta+V$ as an operator sum; all the physics is unchanged.

\section{§ 1. Definition of the Hamiltonian}

The definition and self-adjointness of the Hamiltonian for the $L^{2}$ classes depends on the basic theorem:

Theorem 1 (Kato-Rellich $[3,14])$. Let $H_{o}$ be self-adjoint and suppose $V$ is a symmetric operator with $D(V) \supset D\left(H_{o}\right)$ so that for some $a<1$ and $b$

$$
\|V \phi\| \leqq a\left\|H_{o} \phi\right\|+b\|\phi\|
$$

for all $\phi \in D\left(H_{o}\right)$. Then $H_{o}+V$ defined on $D\left(H_{o}\right) \cap D(V) \equiv D\left(H_{o}\right)$ is selfadjoint. If $H_{o}$ is bounded below, so is $H=H_{o}+V$.

For the Rollnik classes, one needs the analogous theorem:

Theorem 2 (KLMN ${ }^{6}$ theorem). Let $H_{o}$ be a positive self-adjoint operator and suppose $V(\phi, \psi)$ is a symmetric bilinear form with $Q(V)$ $\supset Q\left(H_{o}\right)$ so that for some $a<1$ and $b$,

$$
|V(\phi, \phi)| \leqq a\left\langle\phi, H_{o} \phi\right\rangle+b\|\phi\|^{2}
$$

for all $\phi \in Q\left(H_{o}\right)$. Then the quadratic form $\phi \rightarrow\left\langle\phi, H_{o} \phi\right\rangle+\langle\phi, V \phi\rangle$ defined on $Q\left(H_{o}\right) \cap Q(V) \equiv Q\left(H_{o}\right)$ is the form of a self-adjoint operator, $H$, which is bounded below.

Proof. See Appendix 2. Q.E.D.

${ }^{5}$ E.g. Grossman and $\mathrm{Wu}$ [11] who prove forward dispersion relations for $V \in L^{1} \cap R$ add an $L^{2}$ condition when they wish to worry about foundational questions.

${ }^{6}$ Cannon [15] gives this name to a slightly generalized version of this theorem. The letters stand for Kato, Lions, Lax-Milgram, Nelson [16]. 
We will write $H=H_{o}+V$ in contradistinction to the "usual" convention which reserves " + " for an operator sum.

The KLMN theorem can be used to define the Hamiltonian, for:

Theorem 3. Let $V \in R+L^{\infty}$. Then for any $a<1$, there is a $b$ so that

$$
\langle\phi, V \phi\rangle \leqq a\left\langle\phi, H_{o} \phi\right\rangle+b\|\phi\|^{2}
$$

for all $\phi \in Q\left(H_{o}\right)$ where $H_{o}=-\Delta$. Then $-\Delta+V$ defined as the sum of quadratic forms is the form of a self-adjoint Hamiltonian operator.

Proof ${ }^{7}$. Let $E=k^{2}<0$ and suppose first $V \in R$. Then $V_{\|}^{1 / 2}\left(E+H_{o}\right)^{-1} V_{\|}^{1 / 2}$ is an integral operator with Hilbert-Schmidt norm

$$
\left[\int d x d y(4 \pi)^{-2}|x-y|^{-2} e^{-2 k|x-y|}|V(x)||V(y)|\right]^{1 / 2} .
$$

This goes to 0 as $k \rightarrow \infty$ since $V \in R$. Thus $\left\|\mathrm{V}_{\|}^{1 / 2}\left(E+H_{o}\right)^{-1 / 2}\right\|<a$ if $E$ is taken large enough. For such an $E$, and any $\phi \in Q\left(H_{o}\right)$

For

$$
\begin{aligned}
\langle\phi, V \phi\rangle \mid & \leqq\left\|V_{\|}^{1 / 2} \phi\right\|^{2} \leqq a^{2}\left\|\left(E+H_{o}\right)^{1 / 2} \phi\right\|^{2} \\
& \leqq a^{2}\left\langle\phi, H_{o} \phi\right\rangle+a^{2} E\|\phi\|^{2} .
\end{aligned}
$$

we find

$$
V=W+V_{\infty} ; \quad W \in R ; \quad V_{\infty} \in L^{\infty},
$$

$$
|\langle\phi, V \phi\rangle| \leqq a^{2}\left\langle\phi, H_{o} \phi\right\rangle+\left(a^{2} E+\left\|V_{\infty}\right\|_{\infty}\right)\|\phi\|^{2} \text {. Q.E.D. }
$$

Remarks. 1. There exist $V \in R$ with $D(V) \cap D\left(H_{o}\right)=\{0\}$. Thus, while the Hamiltonian operator we have defined is an extension of the operator sum, it may be defined on a much larger domain!

2. $D(H)$ can be described explicitly. Let $\phi \in Q\left(H_{0}\right)$. It is not hard to prove $-\Delta \phi$ and $V \phi$ both make sense as distributions; $D(H)$ is just those $\phi$ for which $-\Delta \phi+V \phi \in L^{2}$. This can happen when $-\Delta \phi, V \phi \in L^{2}$ but also if there are cancellations.

3. Theorem 2 does not require $V$ to be the form of an operator. For example, in one dimension, $Q\left(-\frac{d^{2}}{d x^{2}}\right)$ contains only bounded continuous functions and for any $a<1,|f(0)|^{2} \leqq a\left\|f^{\prime}\right\|^{2}+b\|f\|^{2}$ for some $b$. Thus $-\frac{d^{2}}{d x^{2}}+\delta(x)$ can be defined as we have defined our Hamiltonian. The cancellations alluded to in Remark 2 can be seen explicitly in this example. A core for $-\frac{d^{2}}{d x^{2}}+\delta(x)$ are $L^{2}$-functions, $f, C^{\infty}$ on $\mathbb{R}-\{0\}$, continuous at 0 with left and right derivatives at 0 with $\phi^{\prime}(0+)-\phi^{\prime}(0-)=\phi(0)$.

${ }^{7}$ We are a little vague about certain technical details involving the meaning of $V_{\|}^{1 / 2}\left(E+H_{o}\right)^{-1 / 2}$, etc. See [5] for these details. 
For $L^{2}$-potentials the formula

$$
(E+H)^{-1}=\left(E+H_{o}\right)^{-1}\left(1+V\left(E+H_{o}\right)^{-1}\right)^{-1}
$$

is often useful. In the Rollnik case, $D(V)$ may not include $\operatorname{Ran}\left(\left(E+H_{o}\right)^{-1}\right)$ $\equiv D\left(H_{o}\right)$, so such an expression may not make sense. However, $\left(E+H_{o}\right)^{-1 / 2} V\left(E+H_{o}\right)^{-1 / 2}$ not only makes sense, it is Hilbert-Schmidt when $V \in R$. This suggests:

Theorem 4. Let $V \in R+\left(L^{\infty}\right)_{\varepsilon}$. Suppose $-E \notin \operatorname{spec}(H)$ where $H=H_{o}+V$ in the forms sense. Then $1+\left(E+H_{o}\right)^{-1 / 2} V\left(E+H_{o}\right)^{-1 / 2}$ has an inverse and ${ }^{8}$ :

$(E+H)^{-1}=\left(E+H_{o}\right)^{-1 / 2}\left[1+\left(E+H_{o}\right)^{-1 / 2} V\left(E+H_{o}\right)^{-1 / 2}\right]^{-1}\left(E+H_{o}\right)^{-1 / 2}$.

Proof. We sketch a proof: for details, see [5]. Take $E$ so negative that $\left[1+\left(E+H_{o}\right)^{-1 / 2} V\left(\mathrm{E}+H_{o}\right)^{-1 / 2}\right]^{-1}$ exists as a geometric series. Treating the various maps as maps between the scale spaces $\mathscr{H}_{ \pm 1}{ }^{9}$ one verifies Tiktopoulos' formula. The extension to all points where the inverse exists follows by analytic continuation. That the inverse exists when $-E \notin \operatorname{spec}(H)$ follows from our discussion in $\S 2$. Q.E.D.

As a typical application of this theorem, we note

Corollary 5. Let $V \in R+\left(L^{\infty}\right)_{\varepsilon}$. Then $H=H_{o}+V$ has only discrete spectrum $^{10}$ in $(-\infty, 0)$.

Proof $^{11} .\left(E+H_{o}\right)^{-1 / 2} V\left(E+H_{o}\right)^{-1 / 2}$ is Hilbert-Schmidt and analytic in the cut plane if $V \in R$. If $V \in R+\left(L^{\infty}\right)_{\varepsilon}$ it is analytic and a limit of HilbertSchmidt operators, hence compact. By a standard theorem [17] on analytic compact families $\left[1+\left(E+H_{0}\right)^{-1 / 2} V\left(E+H_{o}\right)^{-1 / 2}\right]^{-1}$ exists except for a discrete set and is meromorphic with finite rank residues ${ }^{12}$. The theorem follows from Tiktopoulos' formula. Q.E.D.

\section{§ 2. Bound States}

There are two main techniques for carrying over the qualitative theory of bound states from the $L^{2}$ to the Rollnik classes. One is to prove results directly. The second is to approximate $V \in R+\left(L^{\infty}\right)_{\varepsilon}$ by

${ }^{8}$ Such a formula was first suggested to the author by G. Tiktopoulos (private communication) originally as a method for defining $H$. We call this expression Tiktopoulos' formula.

9 See Appendix 2.

10 That is, only isolated eigenvalues of finite multiplicity.

${ }^{11}$ The idea of the proof parallels an idea of Hunziker [4].

12 This theorem also requires the inverse to exist somewhere. Since

$$
\left\|\left(E+H_{o}\right)^{-1 / 2} V\left(E+H_{o}\right)^{-1 / 2}\right\| \rightarrow 0
$$

as $E \rightarrow \infty$, we know this condition is met. 
$V_{n} \in L^{2}+\left(L^{\infty}\right)_{\varepsilon}$ and use the following elementary consequence of Tiktopoulos' formula:

Theorem 6. Let $V_{n}, V \in R+\left(L^{\infty}\right)_{\varepsilon}$ and suppose $\left\|V_{n}-V\right\|_{R} \rightarrow 0$ as $n \rightarrow \infty$. Then for any $E \notin \operatorname{spec} H\left(H=H_{o}+V\right.$ in the form sense).

$$
\left(H_{n}-E\right)^{-1} \rightarrow(H-E)^{-1} \quad \text { (norm topology). }
$$

In particular

(a) $e^{i H_{n} t} \rightarrow e^{i H t} \quad$ (strong topology),

(b) $E_{[a, b]}\left(H_{n}\right) \rightarrow E_{[a, b]}(H)$ (in \|\|$)$

if $a<b<0$ aren't eigenvalues of $H$ and $E_{\Omega}(A)$ is a spectral projection of $A$.

(c) $E_{[a, \infty)}\left(H_{n}\right) \rightarrow E_{[a, \infty)}(H)$ (strong topology)

if $a>0$ and isn't an eigenvalue.

Proof. (a) follows from the norm convergence of the resolvents by the Trotter-Kato theorem [21, p. 511]. (b) and (c) follow by elementary theorems [21, p. 432] and [22, p. 372]. Q.E.D.

Any $V \in R+\left(L^{\infty}\right)_{\varepsilon}$ can be \|\|$_{R}$-approximated by $V_{n} \in L^{2}+\left(L^{\infty}\right)_{\varepsilon}$. We can thus use (b) of Theorem 6 to carry over any bounds on the number of bound states proven for $V \in L^{2}+\left(L^{\infty}\right)_{\varepsilon}$ in particular:

Theorem 7. Let $V \in R+\left(L^{\infty}\right)_{\varepsilon}$. Let $N(V)$ be the number of negative energy bound states. If $V$ is central, let $n_{l}(V)$ be the number of such states of angular momentum $l$ (not counting multiplicity). Then

(a) $\quad N(V) \leqq \int \frac{\theta(-V(x)) \theta(-V(y)) V(x) V(y)}{|x-y|^{2}} d x d y$ (Schwinger [11]),

(b) $N(V) \leqq \int V(x) V(y)|x-y|^{-2} d x d y \quad$ (Ghirardi-Rimini [23]),

(c) $n_{l}(V) \leqq(2 l+1)^{-1} \int r|V(r)| d r \quad$ (Bargmann [24]),

(d) $n_{l}(V) \leqq \frac{2}{\pi} \int|V(r)|^{1 / 2} d r \quad$ (Calogero [25]).

In our study of the Tiktopoulos formula, we have already met the question of when solutions of $\left[V_{\|}^{1 / 2}\left(E-H_{o}\right)^{-1} V^{1 / 2}\right] \psi=\psi$ exist. Formally, this equation is equivalent to $\left(E-H_{o}\right)^{-1} V \phi=\phi$ with $\psi=V_{\|}^{1 / 2} \phi$ and this latter equation is equivalent to $H \phi=E \phi$. These formal equivalences depend on writing $H=H_{o}+V$, and expression which is not true in the operator sense. Nevertheless, a careful argument allows one to prove:

Theorem 8. Let $V \in R+\left(L^{\infty}\right)_{\varepsilon}$ and let $E$ be in the complex plane cut by $[0, \infty)$. Then

$$
V_{\|}^{1 / 2}\left(E-H_{o}\right)^{-1} V^{1 / 2} \psi=\psi
$$


has a solution if and only if

$$
H \phi=E \phi
$$

has a solution $\phi \in D(H)$. The solutions $\phi, \psi$ are related by:

$$
\psi=V_{\|}^{1 / 2} \phi ; \quad \phi=\left(E-H_{o}\right)^{-1} V^{1 / 2} \psi .
$$

In particular, $V_{\|}^{1 / 2}\left(E-H_{o}\right)^{-1} V^{1 / 2}$ cannot have eigenvalue 1 if $\operatorname{Im} E \neq 0$.

Proof. See [5], Section III.2.

This integral equation form of the $\psi$ equation, viz.

$$
-\int d x V_{\|^{1 / 2}}(x)(4 \pi)^{-1} e^{i k|x-y|} V^{1 / 2}(y) \psi(y)=\psi(x)\left[\operatorname{Im} k>0 ; k^{2}=E\right]
$$

can be used to prove Schwinger's bound directly; in fact a careful argument shows 0 energy bound states can be included in the inequality [5]. As Scadron et al. [11] have noted $V_{\|}^{1 / 2}\left(E \pm i 0-H_{o}\right)^{-1} V^{1 / 2}$ are welldefined Hilbert-Schmidt integral operators when $V \in R$ even if $E$ is real and positive. This leads one to suspect an integral equation might hold for positive energy bound states. One does: Then:

Theorem 9. Let $V \in R$ and $E \geqq 0$. Suppose $H \phi=E \phi$ and let $\psi=V_{\|}^{1 / 2} \phi$.

In particular:

$$
\begin{aligned}
\left\{\left[V_{\|}^{1 / 2}(E \pm i 0\right.\right. & \left.\left.\left.-H_{o}\right)^{-1} V^{1 / 2}\right] \psi\right\}(x) \\
& \equiv-(4 \pi)^{-1} \int V_{\|}^{1 / 2}(x) \frac{e^{ \pm i V E|x-y|}}{|x-y|} V^{1 / 2}(y) \psi(y) \\
& =\psi(x) .
\end{aligned}
$$

(a) If $\|V\|_{R}<4 \pi, H$ has no positive bound states.

(b) If $\int e^{A|x|} e^{A|y|}|x-y|^{-2}|V(x)||V(y)| d x d y<\infty$ for some $A>0, H$ has at worst finitely many bound states.

(c) $H$ cannot have positive energy bound states for arbitrary large $E$.

Proof. See [5], Section III.4. Q.E.D.

We note that (b) holds because $V_{\|}^{1 / 2}\left(E+i 0-H_{o}\right)^{-1} V^{1 / 2}$ can be continued to a strip $\operatorname{Im} E>-C$, some $C>0$. By the analytic Fredholm theorem [17], the integral equation only has solutions for discrete $E$. In the general case, a Reimann-Lebesgue lemma argument [26] shows $V_{\|}^{1 / 2}\left(E+i 0<H_{o}\right)^{-1} V^{1 / 2}$ has norm less than 1 for $E$ large and thereby (c) is proven.

Remarks. 1. While the existence of a positive energy eigenvalue implies the existence of a solution of the integral equation, the converse is not always true. For example, if $V$ is a square well with a zero energy $s$-wave resonance, the integral equation has a solution for $E=0$ but $H \phi=0$ has no (square integrable) solutions. Whether a like occurrence can occur for $E>0$ is an open question. 
2. The results (a)-(c) above are very weak. However, unlike the "usual" results on positive energy eigenvalues [27] they require no smoothness conditions. We note, however, that Kato [12] has proven (a) by a method employing time-independent scattering theory.

\section{§ 3. Scattering and the Kato-Birman Theorem}

In studying scattering theory, the first goal must be to establish the existence of the strong limits ${ }^{13} \Omega^{ \pm}=\lim _{t \rightarrow \mp \infty} e^{+i H t} e^{-i H_{o} t}$. In passing we note that Kupsch and Sandas [28] have shown how the Hack-Cook proof [29] of the existence of this limit can be modified to handle "singular" potentials which are $0\left(r^{-1-\varepsilon}\right)$ at $\infty$. While such a result could be applied to a class of Rollnik potentials, we will recover stronger results below.

In addition to the existence of $\Omega^{ \pm}$, one would like to prove $\mathscr{H}_{\text {in }}=\mathscr{H}_{\text {out }}$ where $\mathscr{H}_{\text {in }}=\operatorname{Ran} \Omega^{ \pm}$. This equality is equivalent to unitarity of the $S$-matrix ${ }^{14} S=\left(\Omega^{-}\right)^{*} \Omega^{+}$and we will call this equality weak asymptotic completeness (WAC).

WAC was first proven for $V \in L^{1} \cap L^{2}$ by Kuroda [30] who actually proved the stronger set of equalities $\mathscr{H}_{\text {in }}=\mathscr{H}_{\text {out }}=\mathscr{H}_{\text {a.c. }}$ where $\mathscr{H}_{\text {a.c. }}$ is the space of absolutely continuous vectors for $H^{15}$. Kuroda's completeness proof has been abstracted as follows:

Definition. Let $H_{1}, H_{2}$ be self-adjoint and let $P_{1}, P_{2}$ be the projections onto their absolutely continuous spaces. We say $\Omega^{ \pm}\left(H_{2}, H_{1}\right)$ exist if $\lim e^{+i H_{2} t} e^{-i H_{1} t} P_{1}$ exist. If their ranges are $P_{2} \mathscr{H}$ then we say $\Omega^{ \pm}\left(H_{2}, H_{1}\right)$ $t \rightarrow \pm \infty$ obey (KC) [for Kato-Kuroda completeness].

Theorem 10. (Kato-Birman [31]). If either:

(a) $\mathrm{H}_{2}-\mathrm{H}_{1}$ is trace class or

(b) $\left(H_{2}+E\right)^{-1}-\left(H_{1}+E\right)^{-1}$ is trace class for some $E$ then $\Omega^{ \pm}\left(H_{2}, H_{1}\right)$ exist and are complete.

Proof. See [21], pp. 535-546. Q.E.D.

The Kato-Birman theorem is a precise mathematical form of the general feeling that $\Omega^{ \pm}\left(H_{2}, H_{1}\right)$ should exist if $H_{2}-H_{1}$ is a "gentle" perturbation of $H_{1}$. Physically, a perturbation is "gentle" viz. a viz. scattering if it falls off rapidly at $\infty$; i.e. we expect perturbation by a

${ }^{13}$ We follow the physicist's conversion derived from $\pm i \varepsilon$ equations of calling the limit as $t \rightarrow \mp \infty, \Omega^{ \pm}$.

${ }^{14}$ Contrary to folklore, unitarity is not merely a consequence of conservation of probability.

${ }^{15}$ See [5], Appendix (b), [21], pp. 516-517 or $\S 4$ below for the definition of $\mathscr{H}_{\text {a.c. }}$. 
potential with "bad" finite singularities but which is well behaved at $\infty$ to have little effect on existence questions for scattering. In fact

Theorem 11. Let $V_{1}, V_{2} \in R+L^{\infty}$ and suppose $\Omega^{ \pm}\left(H_{o}+V_{1}, H_{o}\right)$ exist and obey (KC). If $V_{1}-V_{2} \in L^{1} \cap R$, then $\Omega^{ \pm}\left(H_{o}+V_{2}, H_{o}\right)$ exist and obey $(K C)$.

Proof. By a straightforward but gory computation employing Tiktopoulos' formula as starting point one shows $\left(H_{o}+V_{2}+E\right)^{-1}$ $-\left(H_{o}+V_{1}+E\right)^{-1}$ is trace class if $V_{2}-V_{1} \in L^{1} \cap R$ (see [5], Section IV.3). By the Kato-Birman theorem, $\Omega^{ \pm}\left(H_{o}+V_{2}, H_{o}+V_{1}\right)$ exist and obey (KC). The chain rule ([21], p. 532) which is easy to prove says that $\Omega^{ \pm}\left(H_{3}, H_{1}\right)$ exist and obey (KC) if $\Omega^{ \pm}\left(H_{2}, H_{1}\right) ; \Omega^{ \pm}\left(H_{3}, H_{2}\right)$ exist and obey (KC) so the theorem is proven. Q.E.D.

Theorem 11 is an exact form of the fact that only long distance behavior should matter in the existence of scattering. Once someone else has done the hard work of proving existence and unitarity of $S$ for a class of potentials with some behavior at $\infty$ but with restricted behavior at finite points, we can extend the result to that behavior at $\infty$ with arbitrary locally Rollnik finite point behavior. Let us consider some examples:

(1) $V \in L^{1} \cap R$. Take $V_{2}=V ; V_{1}=0$. Conclude in this case that Kuroda's $L^{1} \cap L^{2}$ result extends "easily" to $L^{1} \cap R$.

(2) $V \in R$. Kato [12] has proven that $\Omega^{ \pm}$are unitary equivalences of $H_{o}$ and $H_{o}+W$ if $\|W\|_{R}<4 \pi$. Take $V_{2}=V, V_{1}=V \chi_{X}$ where $\chi_{X}$ is the characteristic function for $\{x|x|>X\}$ and $X$ is chosen so $\left\|V \chi_{X}\right\|<4 \pi$. Then $V_{2}-V_{1}$ is in $R$ and of compact support, thus in $L^{1} \cap R$ so $\Omega^{ \pm}$exist and are complete in this case.

(3) $V \in R+L^{\infty} ; V=0\left(r^{-1-\varepsilon}\right)$ at $\infty$. Kato and Kuroda [32] have proven $(\mathrm{KC})$ for potentials $W$ with $|W(\boldsymbol{r})| \leqq C(1+r)^{-1-\varepsilon}$ for all $\boldsymbol{r}$. Taking $V_{2}=V$ and $V_{1}=V$ at large distances, we obtain $(\mathrm{KC})$ for this class.

(4) $V=\gamma r^{-1}+W ; W \in L^{1} \cap R$. Dollard [33] has shown $\Omega^{ \pm}$don't exist in this case but the following modified scattering theory works. There exists an "approximately free" dynamics $U_{D}(t)$ (dependent on $\gamma$ ) so that $U_{D}(t) f$ and $e^{-i H_{o} t} f$ have identical probability distributions in $x$

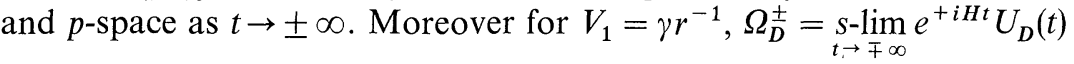
exist and are complete. We conclude that for the $V$ considered above, we have modified wave operators $\Omega_{D}^{ \pm}$which obey $(\mathrm{KC})$.

(5) $V=\gamma r^{-\beta}+W ; 1<\beta<\frac{3}{4} ; W \in L^{1} \cap R$. Amrein, Martin, and Misra [34] have shown a modified dynamics $U_{D}(t)^{16}$ exist in the case $\left(V_{1}=\gamma r^{-\beta}\right)$

${ }^{16}$ Amrein $e t$ al. only show $U_{D}(t) f$ and $e^{-i H_{o} t} f$ have asymptotically identical $p$-space probability distributions, but simple computation shows this is true in $x$-space as well. 
and that $\Omega_{D}^{ \pm}$exist and are complete. We extend their result to $\gamma r^{-\beta}+W$ directly.

Before leaving time-dependent scattering theory, let us discuss the question of continuity of $\Omega^{ \pm}$as a function of $V$. Kuroda [30] proved that $\Omega_{n}^{ \pm} \rightarrow \Omega^{ \pm}$(strongly) if $V_{n} \rightarrow V$ in $L^{1}$ and if $\left\|V_{n}\right\|_{2}$ are bounded (all $\left.V_{n}, V \in L^{1} \cap L^{2}\right)$. Kato [21, p. 551] abstracted this theorem to the following: If $\left(H_{n}+E\right)^{-1} \rightarrow(H+E)^{-1}$ in trace-class norm and if each $\left(H_{n}+E\right)^{-1}$ $-\left(H_{o}+E\right)^{-1}$ is in trace class, then $\Omega_{n}^{ \pm} \rightarrow \Omega^{ \pm}$(strongly). Another gory but straightforward computation ([5], Section IV.4) shows:

Theorem 12. Let $V_{n}, V \in L^{1} \cap R$ and let $V_{n} \rightarrow V$ in both \|\|$_{1}$ and \|\|$_{R}$. Then $\Omega_{n}^{ \pm} \rightarrow \Omega^{ \pm}$(strongly) and $S_{n} \rightarrow S$ (strongly).

Proof. See [5], Section IV.4. We only remark that the strong convergence of the $\Omega$ 's implies weak convergence of the $S$-matrices and unitarity of the $S$-matrices then implies strong convergence. Q.E.D.

Remarks. 1. At first sight Theorem 12 does not appear to generalize Kuroda's result since we require convergence in \|\|$_{R}$ and Kuroda only requires boundedness in \|\|$_{2}$. However, the inequality (appendix 1) $\|V\|_{R} \leqq 3^{1 / 2}(2 \pi)^{1 / 3}\|V\|_{2}^{2 / 3}\|V\|_{1}^{1 / 3}$ shows that convergences in \|\|$_{1}$ and boundedness in \|\|$_{2}$ implies \|\|$_{R}$-convergence. Thus, Theorem 12 does, in fact, generalize Kuroda's result.

2. Like Kuroda's result, Theorem 12 is essentially a theorem in timedependent scattering theory. However, unlike Kuroda's result, it has an immediate interpretation in terms of time-independent scattering theory. For, if we are to accept the fundamental notions of time-independent scattering theory, scattering is described by the on-shell $T$-matrix:

$$
T\left(k, k^{\prime}\right)=(2 \pi)^{-3} \int\left[e^{-i k \cdot x} V^{1 / 2}(x)\right]\left[V_{\|}^{1 / 2}(x) \phi\left(k^{\prime}, x\right)\right] d^{3} x
$$

where $\phi\left(k^{\prime}, x\right)$ is the Lippman-Schwinger in-wave function. As we shall see below (Section 4), $V_{1 / 2}^{1 / 2} \phi$ solves an $L^{2}$-integral equation whose kernel is $V_{\|}^{1 / 2}\left(k^{\prime 2}+i 0-H_{o}\right)^{-1} V^{1 / 2}$ and whose homogeneous term is $V_{\|}^{1 / 2}(x) e^{i k^{\prime} \cdot x}$. Thus $V_{n} \rightarrow V\left(\|\|_{1}\right)$ tells us the inhomogeneous terms converge and $V_{n} \rightarrow V\left(\|\|_{R}\right)$ tells us the first bracket in the $T\left(k, k^{\prime}\right)$ integral converges in $L^{2}$. Thus, modulo technical points and the fact that we have not yet justified the formulae of time-independent scattering theory, we can also prove Theorem 12 in the context of the time-independent theory.

\section{§ 4. Eigenfunction Expansions}

In order to have a physical interpretation for all states of an interacting two-body system, we must demand more than $\mathscr{H}_{\text {in }}=\mathscr{H}_{\text {out }}$. We must also know $\mathscr{H}_{\text {in }}=\mathscr{H}_{\text {out }}=\left(\mathscr{H}_{\text {bound }}\right)^{\perp}$ where $\mathscr{H}_{\text {bound }}$ is the space of 
eigenfunctions for $H$. For physically one expects any state which has no bound component to "decay" asymptotically in the past and future. We will call this stronger condition $\mathscr{H}_{\text {in }}=\mathscr{H}_{\text {out }}=\left(\mathscr{H}_{\text {bound }}\right)^{\perp}$ asymptotic completeness (AC).

To see how much stronger $(\mathrm{AC})$ is than $(\mathrm{KC})$, we recall the definition of singular spectrum. The spectral theorem tells us every self-adjoint operator, $A$, is (unitarily equivalent to) a multiplication operator on $L^{2}$ of some measure on IR. Any measure can be decomposed uniquely into a sum of three pieces: a pure point part (i.e. a measure which is a sum of delta functions), a measure absolutely continuous with respect to Lebesgue measure (i.e. $v(A)=\int_{A} f(x) d x, f$ locally $\left.L^{1}(d x)\right)$ and a continuous singular measure (i.e. a measure, like the Cantor measure, which has no pure points and which lives on a set of Lebesgue measure 0 ). Correspondingly the base Hilbert space $\mathscr{H}$ breaks up into a direct sum $\mathscr{H}_{\text {p.p. }} \oplus \mathscr{H}_{\text {a.c. }}$ $\oplus \mathscr{H}_{\text {sing }}$ so that $A$ leaves each space invariant and $A \vdash \mathscr{H}_{\text {p.p. }}$. has only pure point spectrum (i.e. its spectral measure is a pure point measure), etc. Since $\Omega^{ \pm}$is a unitary equivalence of $H_{o}$ and $H \vdash \mathscr{H}_{\text {in(out) }}, \mathscr{H}_{\text {in(out) }}$ $C \mathscr{H}_{\text {a.c. }}$, so if $(\mathrm{AC})$ holds we must have $\mathscr{H}_{\text {sing }}=0$ and (KC). Conversely $(\mathrm{KC})$ and $\mathscr{H}_{\text {sing }}=0$ implies (AC).

In the two-body case, Ikebe [35] has proven (AC) for Hölder continuous $V$ which are $0\left(r^{-2-\varepsilon}\right)$ at $\infty$. His proof depends crucially on the existence of an eigenfunction expansion for $H$, i.e. a generalized Fourier transform with $e^{i k \cdot x}$ replaced by an "eigenfunction" for $H$. Such an expansion is of great interest in and of itself. First, it gives us an explicit handle on $H$. Secondly, since the eigenfunctions can be chosen as Lippmann-Schwinger wave functions, this approach provides the connection between time dependent and time independent scattering theory.

The eigenfunction expansion with which Ikebe deals is not merely an expansion in an abstract rigged Hilbert space setting. The eigenfunctions are constructed in $x$-space as perturbations of the $H_{o}$ eigenfunctions. As such, they allow comparison of various spectral properties of $H$ and $H_{o}$. On the other hand, a rigged Hilbert space expansion of the simplest type deals only with $H$ : since the spectral theorem says $H$ is a multiplication operator on some $L^{2}$-space, it is easy to realize $H$ as a multiplication operator on a distribution space with $\delta$-functions as eigenfunctions. Thus, such an eigenfunction expansion is essentially a form of the spectral theorem and as such cannot restrict spectral properties. More complicated riggings usually require strong conditions on $H$ and $H_{o}$ such as the existence of a dense domain for $H^{n} H_{o}^{m}$ (each $\left.n, m\right)$. In the more prosaic approach of Ikebe, no such conditions are needed.

In this section, we develop an Ikebe-type eigenfunction expansion for arbitrary $V \in L^{1} \cap R$. In case $V$ has exponential falloff, we can use this 
expansion to prove $\mathscr{H}_{\text {sing }}=0$ and thus also (AC). Our proof essentially follows that of Ikebe with three crucial differences: (1) We do not need to introduce any auxiliary spaces; by using the trick of "factorized" integral equations, we only deal with objects in $L^{2}$. We thus need no smoothness assumptions but as a result can only treat $V \in L^{1}$ rather than $0\left(r^{-2-\varepsilon}\right)$. (2) We are only able to prove (AC) under the strong condition that $V$ falls off exponentially. However, we provide a new proof of (KC). More importantly, we do not require any a priori information on the nonexistence of positive energy bound states (pebs) to obtain this weak result. In contrast, Ikebe depends on a result of Kato on pebs. In the manybody case, Hepp's results [36] are weakened because he too must first show no pebs exist and no strong results on pebs exist in the many-body case. It is our hope that an analogue of the proof we give here will enable one to prove (AC) in the many-body case without any a priori knowledge of pebs. (3) We correct a minor error (described in detail in [5], Section V.2) in Ikebe's proof. Ikebe ${ }^{17}$ has found an alternate way of correcting this error $^{18}$.

We start with the formal Lippman-Schwinger equation $\phi=e^{i k \cdot x}$ $+\left(E+i 0-H_{o}\right)^{-1} V \phi$. Letting $\psi=V_{\|}^{1 / 2} \phi$, we see

$$
\psi=V_{\|}^{1 / 2} e^{i k \cdot x}+V_{\|}^{1 / 2}\left(E+i 0-H_{o}\right)^{-1} V^{1 / 2} \psi .
$$

As long as the kernel has no homogeneous solution, we can solve for $\psi$ (and thus $\phi$ ) since the kernel is Hilbert-Schmidt and the inhomogeneous term is $L^{2}$. The crucial fact is:

Theorem 13. Let $\mathscr{E}$ be the set of real positive $E$ for which

$$
V_{1 /}^{1 / 2}\left(E+i 0-H_{o}\right)^{-1} V^{1 / 2} \eta=\eta
$$

has an $L^{2}$-solution $\eta$. Then:

(a) If $V \in R \cap L^{1}, \mathscr{E}$ is a closed set of Lebesgue measure 0 .

(b) If $\|V\|_{R}<4 \pi, \mathscr{E}=\phi$.

(c) If $\int e^{A|x|} e^{A|y|}|x-y|^{-2}|V(x)||V(y)| d x d y<\infty$ for some $A>0$, then $\mathscr{E}$ is a finite set.

Proof. (a) The kernel in question is the boundary value of a kernel analytic in the cut plane continuous on the two lips of the cut. By a modified Fredholm argument $\mathscr{E}$ is the set of zeros of a function analytic in the upper half-plane, continuous on the lips. By a general theorem [37], $\mathscr{E}$ is closed of measure 0.

(b) In this case, $\left\|V_{\|^{1 / 2}}\left(E+i 0-H_{o}\right)^{-1} V^{1 / 2}\right\|<1$.

${ }^{17} T$, Ikebe, private communication and work in preparation.

${ }^{18}$ However, Ikebe's correction utilizes smoothness and won't carry over to the $L^{1} \cap R$ case we consider.

15 Commun. math. Phys., Vol 21 
(c) In this case, the kernel extends onto a second sheet so $\mathscr{E}$ is the zeros of an analytic function extendable to a second sheet. By a KleinZemach argument [26] $\mathscr{E}$ is bounded, so it is finite. Q.E.D.

We can now establish the eigenfunction expansion.

Theorem 14. Let $\phi(x, k)$ be the Lippmann-Schwinger eigenfunctions defined for $k^{2} \notin \mathscr{E}$, all $x$. Then ${ }^{19}$

(a) $\hat{f}(k)=1$. i.m. $(2 \pi)^{-3 / 2} \int \overline{\phi(x, k)} f(x) d x$ exist for all $f \in L^{2}$.

(b) 1.i.m. $(2 \pi)^{-3 / 2} \int \phi(x, k) \hat{f}(k) d k$ exists and equals $\left(E_{\text {a.c. }} f\right)(x)$ where $E_{\text {a.c. }}$ is the projection onto $\mathscr{H}_{\text {a.c. }}$.

(c) Let $[\alpha, \beta] \cap \mathscr{E}=\phi$, with $0 \leqq \alpha<\beta$. Then

$$
\left\|E_{[\alpha, \beta]} f\right\|^{2}=\int_{\alpha<k^{2}<\beta}|\hat{f}(k)|^{2} d k
$$

where $E_{[\alpha, \beta]}$ is a spectral projection for $H$.

(d) $f \in D(H)$ if and only if $E_{\text {sing }} f \in D(H)$ and $\int|k|^{4}|\hat{f}(k)|^{2} d k<\infty$. In that case $\widehat{H f}(k)=k^{2} \hat{f}(k)$.

(e) $\wedge$ is a unitary map of $\mathscr{H}_{\text {a.c. }}$ and $L^{2}(k)$ i.e. ${ }^{\wedge}\left({ }^{\wedge}\right)^{*}=1$ (i.e. ${ }^{\wedge}$ is onto) and $(\hat{\wedge})^{* \wedge}=E_{\text {a.c. }}$ (i.e. (b)).

Proof. See [5], Sections IV.5 and V.4. We remark that the crucial idea [13] is to relate $\phi$ to a boundary value of the Fourier transform of the interacting Green's function. We also note that the proofs of (e) and (d) depend on the scattering theory connection discussed below (Theorem 16) and, in particular, depend on the existence of the wave operators. Q.E.D.

Remark. This result is more or less provable also using new and general methods of Koroda [38].

By (c), the spectrum of $H$ is absolutely continuous in $[\alpha, \beta]$. Since $[\alpha, \beta]$ is arbitrary except for its disjointness from $\mathscr{E}$, we conclude $\sigma_{\text {sing }} \subset \mathscr{E}$. Since $\sigma_{\text {sing }}$ must support a measure with no pure points if it is non-empty, $\sigma_{\text {sing }}$ must be uncountable or empty. We thus conclude:

Theorem 15. Let $V \in L^{1}$. If $\|V\|_{R}<4 \pi, \sigma_{\text {sing }}=\phi$. Or, if

$$
\int|V(x)||V(y)| e^{A|x|} e^{A|y|}|x-y|^{2} d x d y<\infty
$$

for some $A>0$, then $\sigma_{\text {sing }}=\phi$. In both cases, $(A C)$ holds.

Remark. By a different but related method, Kato [12] has proven (AC) when $\|V\|_{R}<4 \pi$ without the extra $V \in L^{1}$ condition. See also [44].

${ }^{19}$ For $x$-space integrals, 1.i.m. means the $L^{2}(k)$ limit as $R \rightarrow \infty$ of the integral over $\{x|| x \mid<R\}$. For $k$-space integrals, 1.i.m. means the $L^{2}(x)$ limit as $R \rightarrow \infty, \varepsilon \rightarrow 0$ of the integral over $\{k|| k \mid<R ; \operatorname{dis}(k, \mathscr{E})<\varepsilon\}$. 
The eigenfunctions $\phi$ that we have used were not chosen at random; they are the Lippmann-Schwinger in-functions. As a result we expect $\Omega^{+}$to take $\phi(x, k)$ into $e^{i k \cdot x}$ in some sense. The link between ${ }^{\wedge}$ and scattering is provided by:

Theorem 16. Let $V \in L^{1} \cap R$. Let $\hat{f}_{0}(k)=(2 \pi)^{-3 / 2}$ 1.i.m. $\int f(x) e^{-i k \cdot x}$ be the ordinary Fourier transform. Then:

(a) $\widehat{\Omega^{+} f}=\hat{f}_{0}$.

(b) If $f, g$ are such that $\hat{f}_{0}, \hat{g}_{0}$ are $C^{\infty}$ of compact support so that all $k^{2} \in \mathscr{E}$ are disjoint from the supports (such f's and g's are dense), then:

$$
\langle f,(S-1) g\rangle=-(2 \pi i) \int d^{3} k d^{3} k^{\prime} T\left(k, k^{\prime}\right) \delta\left(k^{2}-k^{\prime 2}\right) \overline{\hat{f}_{0}(k)} \hat{g}_{0}(k)
$$

where

$$
T\left(k, k^{\prime}\right)=(2 \pi)^{-3} \int e^{-i k \cdot x} V(x) \phi\left(x, k^{\prime}\right) d \chi .
$$

Proof. See [5], Sections V.3-V.5. Q.E.D.

Remarks. 1. For Hölder continuous $V$ of $0\left(r^{-2-8}\right)$ at $r=\infty$, Ikebe $[13,39]$ has proven similar results to those of Theorem 16 . Hunziker [40] has discussed results of the form of Theorem 16 for the $L^{1} \cap R$ case we consider, but without technical details.

2. (a) is used in proving $\wedge$ is onto (Theorem 14(e)). It depends on the fact that $\Omega^{+}$exists but does not require (KC). (a) and Theorem 14(b) provide and alternate proof of $(\mathrm{KC})$. There is little hope of a KatoBirman theorem proof of $(\mathrm{KC})$ extending to a multi-channel $n$-body case, but a proof of $(\mathrm{KC})$ via eigenfunction expansions may extend.

3. Theorem 16 is basic to the rigorous study of "advanced" topics in scattering theory for it provides the connection between first principles time-dependent scattering theory and the useful formulae of timeindependent scattering theory, a connection which is usually sloughed over in the physics literature.

On the basis of Theorem 16, one can present a "from-first-principles" proof of various dispersion relations. Using the method of Grossman-Wu [11], one can prove forward dispersion relations for $V \in L^{1} \cap R^{20}$. The only change from the Grossman-Wu result and proof involves the set $\mathscr{E}$. So far as we know, one can only prove $T(k, k)$ is the boundary value of an analytic function for $k^{2} \notin \mathscr{E}$. In dispersion integrals "Im $f$ " must be viewed as a distribution which is a continuous function (equal to $\operatorname{Im} f$ !) on $\mathbb{R}-\mathscr{E}$.

Using methods of Hunziker [41] and Grossman-Wu [11], one can prove non-forward dispersion relations and analyticity in a Lehmann ellipse when $V$ has exponential fall-off. In this case, one can show the

${ }^{20}$ G. Tiktopoulos, private communication and [5], Chap. VI. 
possible a priori singularities at $k^{2} \in \mathscr{E}$ are removable. The basic idea is as follows: in the case of exponential fall-off the scattering amplitude can be continued to a strip below the real axis. By a Fredholm theory argument, the only singularities allowed are poles. Using Lehmann ellipse analyticity, one obtains partial wave amplitudes with the same energy analyticity properties as the full amplitude. Partial wave unitarity doesn't allow poles in the partial wave amplitudes and thereby in the full amplitude ${ }^{21}$.

Acknowledgments. It is a pleasure to thank many individuals for their aid and suggestions. George Tiktopoulos first pointed out to me the gap between the $L^{2}$ classes and physically singular potentials. He also suggested the $L^{1} \cap R$ class since this class obeys forward dispersion relations. Ed Nelson taught me that it was honorable to define $H_{o}+V$ as a sum of forms. I have profited from discussions with V. Bargmann, C. Fefferman, J. Kohn, M. Reed, and E. Stein and from valuable correspondence with T. Kato and T. Ikebe. Finally, I should like to especially thank A. S. Wightman for his wisdom, encouragement and constructive criticism.

\section{Appendix 1}

\section{The Rollnik Condition}

In this appendix, we wish to discuss the relation of the Rollnik condition to various other and related conditions. First, we note the connection to the $L^{p}$ spaces:

Proposition 1. $L^{3 / 2} C R$.

Proof. Follows from the Sobolev inequality [42].

From Proposition 1 follows the following lattice of containment:

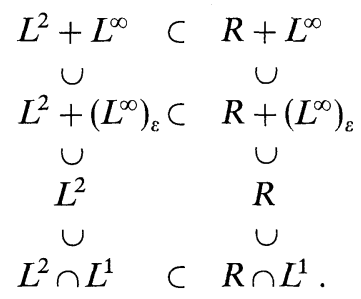

Remarks. 1. Note that $L^{2} C R$ does not hold.

2. Think of the lattice as follows. As one goes up, worse behavior at $\infty$ is allowed. As one goes to right, worse behavior at finite points is allowed.

21 The fact that the position of the poles is independent of angle is critical in this proof. See [5], Section V.4. 
3. Not only is $L^{2} \cap L^{1} \subset R$; one can prove

([5], Section I.1).

$$
\|V\|_{R} \leqq 3^{1 / 2}(2 \pi)^{1 / 3}\|V\|_{2}^{2 / 3}\|V\|_{1}^{1 / 3}
$$

Secondly, we consider the $p$-space forms of $R$ :

Proposition 2. Let $V \geqq 0$. Then $V \in R$ if and only if $\int \frac{|\hat{V}(p)|^{2}}{p} d^{3} p<\infty$ $\left(\hat{V}\right.$ is the ordinary Fourier transform ${ }^{22}$ ). In any event if $V \in R$,

$$
\int p^{-1}|\hat{V}(p)|^{2} d^{3} p<\infty \text { and } \int d^{3} p d^{3} q p^{-2} q^{-2}|\hat{V}(p-q)|^{2}<\infty .
$$

For pathological $V$ which wiggle a lot one can have

$$
\int|\hat{V}(p)|^{2} p^{-1} d^{3} p<\infty
$$

without $V \in R$. Pathologies of this sort and of potentials with singularities on "thin" sets prevent various other relations from always holding so we can only state our final result for monotonic central potentials. As preparation let us define or recall several conditions:

(CB) (for "convergence of Born series"). The integrals $B_{n}=\int\left|V\left(x_{1}\right)\right|\left|x_{1}-x_{2}\right|^{-1} \ldots\left|x_{n-1}-x_{n}\right|^{-1}\left|V\left(x_{n}\right)\right| d x_{1} \ldots d x_{n}<\infty \quad(n=1, \ldots)$ and $\sum_{n=1}^{\infty} \lambda^{n} B_{n}$ has a non-zero radius of convergence.

$\left(W^{p}\right)$ (weak $L^{p}$ ). A measurable function $f$ has a distribution function $m_{f}(t)=\mu(\{x|| f(x) \mid>t\})$. We say $f \in W^{p}$ if $m_{f}(t)<C t^{-p}$ for some $C$ and all $t$.

(KLMN small). We say $A<B(\mathrm{KLMN})$ if $Q(A) \supset Q(B)$ and

$$
|\langle\psi, A \psi\rangle|\langle a|\langle\psi, B \psi\rangle|+b\langle\psi, \psi\rangle
$$

for some $a$ and $b$.

Proposition 3. Let $V(r)$ be central and monotone and consider the conditions:

(a) $V$ obeys (CB),

(b) $V \in W^{3 / 2} \cap L^{1}$,

(c) $V \in L^{1}$ and $V<-\Delta(K L M N)$,

(d) $V \in L^{1}$ and $Q(V) \supset Q(-\Delta)$,

(e) $V \in L^{1}$ and $V_{\|}^{1 / 2}\left(E+H_{o}\right)^{-1} V_{\|}^{1 / 2}$ is bounded for some $E>0$,

(f) $V \in L^{1}$ and $|V(r)| \leqq C r^{-2}+D$ for some $C, D$.

(g) (Khuri Conditions) $\int_{0}^{\infty} r|V(r)| d r<\infty ; \int_{0}^{\infty} r^{2}|V(r)| d r<\infty$,

22 We have changed conventions from Section 4. 
(h) $V \in L^{1} \cap L^{3 / 2}$,

(i) $V \in R \cap L^{1}$.

Then (a), (b), (c), (d), (e), (f) are equivalent and (g) $\Rightarrow(\mathrm{h}) \Rightarrow(\mathrm{i}) \Rightarrow(\mathrm{a})-(\mathrm{f})$. For arbitrary $V$, we have (Scadron et al. [11]) (i) $\Rightarrow$ (a).

\section{Proof. See [5], Chapter I. Q.E.D.}

(g), (h), (i) and (a)-(f) differ only logarythmically in the central monotone case. The potential $V_{\alpha}(r)=r^{-2}(\log r)^{-\alpha}$ for small $r$ (say $V$ vanishes if $r>e^{-1}$ ) has $V_{\alpha}$ obey (g) if and only if $\alpha>1 ; V_{\alpha}$ obeys (h) if and only if $\alpha>2 / 3 ; V_{\alpha}$ obeys (i) if and only if $\alpha>1 / 2$ and obeys (a)-(f) if and only if $\alpha \geqq 0$.

\section{Appendix 2}

\section{The KLMN Theorem}

Since it is so basic to our whole approach, we present, for the reader's con venience, a sketch of the proof of the KLMN theorem. Even though this theorem looks similar to the Kato-Rellich theorem (Theorem 1) its proof is completely different and is related to the proofs of the Freidrichs extension [18] particularly the proof of Freudenthal [19] (see also [16]).

Let $H_{o}$ be self-adjoint and positive. Norm $Q\left(H_{o}\right)$ with the norm $\|\psi\|_{+1}=\left\langle\psi,\left(H_{o}+1\right) \psi\right\rangle$. $Q\left(H_{o}\right)$ then becomes a Hilbert space, $\mathscr{H}_{+1}$. Suppress the usual identification of $\mathscr{H}_{+1}$ and its dual, $\mathscr{H}_{-1}$, and instead imbed $\mathscr{H}_{+1}$ in $\mathscr{H}_{-1}$ by associating $\psi \in \mathscr{H}_{+1}$ with the functional $\phi \rightarrow\langle\psi, \phi\rangle$ (inner product in $\mathscr{H}$, not $\left.\mathscr{H}_{+1}\right)$. Thereby $\mathscr{H}_{+1} \subset \mathscr{H} \subset \mathscr{H}_{-1}$. The norm on $\mathscr{H}_{-1}$ is $\|\psi\|_{-1}=\left\langle\psi,\left(H_{o}+1\right)^{-1} \psi\right\rangle(\text { for } \psi \in \mathscr{H})^{23}$.

Proposition 4. Let $A: \mathscr{H}_{+1} \rightarrow \mathscr{H}_{-1}$ be a bijection which is symmetric, i.e. $\langle\phi, A \psi\rangle=\langle A \phi, \psi\rangle$ for all $\phi, \psi \in \mathscr{H}_{+1}$. Let

$$
D(\hat{A})=\left\{\phi \in \mathscr{H}_{+1} \mid A \phi \in \mathscr{H}\right\} .
$$

Then $\hat{A}=A \Gamma D(\hat{A})$ is a self-adjoint operator on $\mathscr{H}$.

Proof. Consider $A^{-1} \Gamma \mathscr{H}: \mathscr{H} \rightarrow D(\hat{A})$. It is an everywhere defined symmetric operator; hence, it is self-adjoint. Since it has no kernel, $\hat{A}=\left(A^{-1}\lceil\mathscr{H})^{-1}\right.$ is self-adjoint.

Proof of Theorem 2. By using a modified $\|\psi\|_{+1}=\left\langle\psi,\left(H_{o}+E\right) \psi\right\rangle$, we see $|V(\phi, \phi)| \leqq a\|\phi\|_{+1}^{2}$. A Cauchy-Schwartz argument shows $|V(\phi, \psi)|$ $\leqq a\|\psi\|_{+1}\|\phi\|_{+1}$. Using this, one shows $H_{o}+V+E$ is a bijection of $\mathscr{H}_{+1} \rightarrow \mathscr{H}_{-1}$ which is symmetric. Thus $H_{o}+V$ on the domain $D(H)$ $=\left\{\psi \mid\left(H_{o}+V\right) \psi \in \mathscr{H}\right\}$ is self-adjoint.

${ }^{23}$ For $H_{o}=-\Delta$, this "scale of spaces" is just the beginning of the chain of ordinary Sobolev spaces. 


\section{References}

1. Von Neumann, J.: Mathematische Grundlagen der Quantenmechanik. Berlin: Springer 1932.

Mackey, G.W.: The mathematical foundation of quantum mechanics. New York: Benjamin 1963.

Jauch, J: Foundations of quantum mechanics. Reading, Massachusetts: AddisonWesley 1968.

Piron, C.: Helv. Phys. Acta 37, 439 (1964).

2. Kato, T.: Progr. Theoret. Phys. (Kyoto) Suppl. 40, 3 (1967).

3. - Trans. Am. Math. Soc. 70, 195 (1951).

4. Hunziker, W.: Helv. Phys. Acta 39, 451, (1966).

5. Simon, B.: Quantum mechanics for Hamiltonians defined as quadratic forms. To be published by Princeton University Press, Princeton, N.J.

6. Landau, L., Lifschitz, E.: Quantum mechanics. Oxford: Pergamon, 1958.

7. Nelson, E.: J. Math. Phys. 5, 332 (1964).

8. Green, T. A., Lanford, O.E.: J. Math. Phys. 1, 131 (1960).

Kuroda, S. T.: J. Math. Phys. 3, 933 -935 (1962).

Khuri, N.: Phys. Rev. 107, 1148 (1957).

9. Strichartz, R.: J. Math. Mech. 16, 1031-1060 (1967).

10. Faris, W.: Pacific J. Math. 22, 47 (1967).

Nelson, E.: Topics in dynamics. Princeton, N.J.: Princeton Univ. Press 1969.

11. Rollnik, H.: Z. Physik 145, 639 (1956).

Schwinger, J.: Proc. Natl. Acad. Sci. U.S. 47, 122 (1961).

Scadron, M., Weinberg, S., Wright, J.: Phys. Rev. 135, B 202 (1964).

Grossman, A., Wu, T.T.: J. Math. Phys. 2, 710 (1961).

12. Kato, T.: Math. Ann. 162, 258 (1966).

Kuroda, S. T.: Sûgaku 18, 74-137 (1966).

Coester,F.: Phys. Rev. 133, B 1516 (1964).

13. Ikebe, T.: Arch. Rat. Mech. Anal. 5, 1 (1960).

14. Rellich, F.: Math. Ann. 113, 600 (1937); 116, 55 (1939); 117, 356, (1940); 118, 462 (1942).

15. Cannon, J.: Princeton Thesis (1968), unpublished.

16. Kato, T.: Univ. of Calif. Technical Report No. 9 (1955), unpublished.

Lions, J.: Equations differentielles operationnelles. Berlin-Göttingen-Heidelberg: Springer 1961.

Lax, P.D., Milgram, A. N.: In: Ann. Math. Study No. 33, Princeton Univ. Press 1954. Nelson, E.: Lecture notes on operator differential euqations (1965), unpublished.

- J. Math. Phys. 5, 1190 (1964), Appendices.

17. Dunford, N., Schwartz, J.: Linear operators. New York: Interscience 1963.

18. Freidrichs, K.: Math. Ann. 109, 465, 685 (1934); 110, 777 (1935).

19. Freudenthal, H.: Proc. Acad. Sci. Amsterdam 39, 832 (1936).

20. Courant, R., Hilbert, D.: Methods of mathematical physics, Vol. I. New York: Interscience 1953.

21. Kato, T.: Perturbation theory for linear operators. Berlin-Göttingen-Heidelberg: Springer 1961.

22. Riesz, F., Nagy, R: Functional analysis. Ungar 1955.

23. Ghirardi, G, Rimini, A.: J. Math. Phys. 6, 40 (1965).

24. Bargmann, V.: Proc. Natl. Acad. Sci. U.S. 38, 961 (1952).

25. Calogero, F.: Commun. Math. Phys. 1, 80 (1965).

26. Zemach, C., Klein, A.: Nuovo Cimento 10, 1078 (1958).

Newton, R.: Scattering theory of waves and particles, p. 284. New York: McGrawHill 1966. 
27. Kato, T.: Commun. Pure Appl. Math. 12, 403 (1959).

Odeh, F.: Proc. Am. Math. Soc. 16, 363 (1965).

Simon, B.: Commun. Pure Appl. Math. 22, 531 (1969).

Weidmann, J.: Bull. Am. Math. Soc. 73, 452 (1967).

— Math. Z. 98, 268 (1967).

Agmon, S.: In: Differential equations, II. Aziz, ed. Princeton, N. J.: Van Nostrand 1969.

28. Kupsch, J., Sandas, W.: Commun. math. Phys. 2, 147 (1966).

29. Cook, J. M.: J. math. Phys. 36, 82 (1957).

Hack, M. N. : Nuovo Cimento 9, 731 (1958).

30. Kuroda, S. T.: Nuovo Cimento 12, 431 (1959).

31. Kato, T.: Proc. Japan Acad. 33, 239 (1957).

Birman, S. Y.: Soviet Math. Dokl. 3, 408 (1962).

32. Kato, T., Kuroda, S. T.: In: Functional analysis and related fields. Berlin, Heidelberg, New York: Springer 1969.

33. Dollard, J.: J. Math. Phys. 5, 729 (1964).

34. Amrein, W., Martin, Ph., Misra, B.: To appear in Helv. Phys. Acta.

35. Povzner, A.: Mat. Sbornik 32, 109 (Russian) (1953).

36. Hepp, K.: Helv. Phys. Acta 42, 425 (1969).

37. Hoffman, K.: Banach spaces of analytic functions. Englewood Cliffs, N. J.: PrenticeHall 1962.

38. Kuroda, S. T.: J. Anal. Math. 20, 57 (1967).

39. Ikebe, T.: Pacific J. Math. 15, 511 (1965).

40. Hunziker, W.: In: Lectures in theoretical physics, Vol. X-A. New York: Gordon and Breach 1968.

41. - Helv. Phys. Acta 34, 593 (1961).

42. Sobolev, S. L.: Recuril Math. 46, 471 (1938).

Stein, E. M., Weiss, G.: J. Math. Mech. 8, 263 (1959).

43. Brownell, F.: Ann. Math. 54, 554 (1951).

44. Rejto, P.: J. Math. Anal. 17, 435 (1967).

Barry Simon

Fine Hall

Princeton University

Princeton, New Jersey 08540, USA 